\title{
OSCILLATION THEOREMS FOR SECOND ORDER LINEAR DIFFERENTIAL EQUATIONS WITH DAMPING
}

\author{
JURANG YAN
}

\begin{abstract}
In this paper, we present some criteria for the oscillation of the differentiäl equation with damping

$$
\left(\gamma(t) x^{\prime}(t)\right)^{\prime}+p(t) x^{\prime}(t)+q(t) x(t)=0, \quad t \in\left[t_{0}, \infty\right),
$$

where $p(t)$ and $q(t)$ are allowed to change sign on $\left[t_{0}, \infty\right)$, and $\gamma(t)>0$. One of our results is new even for the differential equations

$$
x^{\prime \prime}(t)+q(t) x(t)=0,
$$
\end{abstract}

and

$$
x^{\prime \prime}(t)+p(t) x^{\prime}(t)+q(t) x(t)=0 .
$$

In this paper, we study the oscillatory behavior of the solution of the second order differential equation with damping

$$
\left(\gamma(t) x^{\prime}(t)\right)^{\prime}+p(t) x^{\prime}(t)+q(t) x(t)=0,
$$

where $\gamma, p$ and $q$ are continuous on $\left[t_{0}, \infty\right), t_{0}>0, \gamma>0$, and $p$ and $q$ are allowed to take on negative values for arbitrarily large $t$. The oscillatory character is considered in the usual sense, i.e., a solution of equation (1) is said to be oscillatory if it has arbitrarily large zeros; otherwise it is said to be nonoscillatory. Equation (1) is called oscillatory if all its solutions are oscillatory.

In the absence of damping, there is a very large body of literature devoted to the corresponding equations

$$
\begin{gathered}
x^{\prime \prime}(t)+q(t) x(t)=0, \\
\left(\gamma(t) x^{\prime}(t)\right)^{\prime}+q(t) x(t)=0 .
\end{gathered}
$$

Although (1) can be put in the forms (*) and (**) by multiplication by an integrating factor and, if necessary, by simple transformations, there are advantages in obtaining direct oscillation theorems for (1): besides the obvious practical advantage of eliminating the need for the integrating factor, there is an incentive in developing methods which will generalize to more general equations.

The use of averaging functions in the study of oscillation dates back to works of Wintner [8] and Hartman [2]. Coles [1] and Willett [7], and more recently, Kwong and Zettl [4] developed averaging techniques and, respectively, established more general theorems for equations $(*)$ and $(* *)$ by considering weighted averages of the integral of $q$.

Recently, by exploiting more fully a simple "completing square" and averaging technique of Kamenev [3], the author [9] has given the following oscillation theorem for equation (1) with $\gamma(t) \equiv 1$.

Received by the editors August 23, 1984 and, in revised form, September 23, 1985.

1980 Mathematics Subject Classification. Primary 34C10, 34C15.

Key words and phrases. Second order differential equation with damping, oscillation. 
THEOREM. If there exist $\alpha \in(1, \infty)$ and $\beta \in[0,1)$ such that

(3) $\lim _{t \rightarrow \infty} \sup t^{-\alpha} \int_{t_{0}}^{t}[(t-\tau) p(\tau) \tau+\alpha \tau-\beta(t-\tau)]^{2}(t-\tau)^{\alpha-2} \tau^{\beta-2} d \tau<\infty$,

then (1) is oscillatory.

Of particular interest, therefore, is the problem of finding criteria for the oscillation of (1) when (2) or (3) is not satisfied. In this paper we will establish two oscillation theorems. The first theorem considerably improves some known results and the second is new even for equations (*) and (**). Our results are as follows:

THEOREM 1. Suppose that there exist a positive continuously differentiable function $h(t)$ on $\left[t_{0}, \infty\right)$ and a constant $\alpha \in(0, \infty)$ such that

(4) $\lim _{t \rightarrow \infty} \sup t^{-\alpha} \int_{t_{0}}^{t}\left\{(t-\tau)^{\alpha} h(\tau) q(t)\right.$

$$
\begin{aligned}
&-\frac{1}{4}\left[(t-\tau) \frac{h(t) p(t)}{\gamma(t)}+\alpha h(\tau)\right.\left.-(t-\tau) h^{\prime}(\tau)\right]^{2} \\
&\left.\times(t-\tau)^{\alpha-2} \frac{\gamma(\tau)}{h(\tau)}\right\} d \tau=\infty,
\end{aligned}
$$

then equation (1) is oscillatory.

PROOF. Let $x(t)$ be a nonoscillatory solution of (1). Without loss of generality, we suppose that $x(t) \neq 0$ for $t \geq t_{0}$. Furthermore, we put

$$
\omega(t)=\gamma(t) x^{\prime}(t) / x(t) .
$$

Then it follows from (1) that

$$
\omega^{\prime}(t)+\omega^{2}(t) / \gamma(t)+p(t) \omega(t) / \gamma(t)+q(t)=0, \quad t \geq t_{0},
$$

and consequently, for all $t>s \geq t_{0}$,

$$
\begin{aligned}
\int_{0}^{t}(t-\tau)^{\alpha} h(\tau) \omega^{\prime}(\tau) d t+\int_{s}^{t}(t-\tau)^{\alpha} \frac{h(\tau) \omega^{2}(\tau)}{\gamma(\tau)} d \tau \\
+\int_{0}^{t}(t-\tau)^{\alpha} \frac{h(\tau) p(\tau) \omega(\tau)}{\gamma(\tau)} d \tau+\int_{s}^{t}(t-\tau)^{\alpha} h(\tau) q(\tau) d \tau=0
\end{aligned}
$$

Since

$$
\begin{aligned}
\int_{0}^{t}(t-\tau)^{\alpha} h(\tau) \omega^{\prime}(\tau) d \tau= & \alpha \int_{0}^{t}(t-\tau)^{\alpha-1} h(\tau) \omega(\tau) d \tau \\
& -\int_{s}^{t}(t-\tau)^{\alpha} h^{\prime}(\tau) \omega(\tau) d \tau-\omega(s)(t-s)^{\alpha} h(s)
\end{aligned}
$$


we obtain that

$$
\begin{aligned}
& \int_{s}^{t}(t-\tau)^{\alpha} h(\tau) q(\tau) d \tau \\
&=(t-s)^{\alpha} h(s) \omega(s)-\int_{s}^{t} \frac{(t-\tau)^{\alpha} h(\tau) \omega^{2}(\tau)}{\gamma(\tau)} d \tau \\
&-\int_{s}^{t}\left[(t-\tau) \frac{h(\tau) p(\tau)}{\gamma(\tau)}+\alpha h(\tau)-(t-\tau) h^{\prime}(\tau)\right](t-\tau)^{\alpha-1} \omega(\tau) d \tau
\end{aligned}
$$

and hence

$$
\begin{aligned}
& \int_{s}^{t}\left\{(t-\tau)^{\alpha} h(\tau) q(\tau)\right. \\
& \left.\quad-\frac{1}{4}\left[(t-\tau) \frac{h(\tau) p(\tau)}{\gamma(\tau)}+\alpha h(\tau)-(t-\tau) h^{\prime}(\tau)\right]^{2}(t-\tau)^{\alpha-2} \frac{\gamma(\tau)}{h(\tau)}\right\} d \tau \\
& =(t-s)^{\alpha} h(s) \omega(s)-\int_{s}^{t}\left\{(t-\tau)^{\alpha / 2}\left(\frac{h(\tau)}{\gamma(\tau)}\right)^{1 / 2} \omega(\tau)\right. \\
& \quad+\frac{1}{2}\left[(t-\tau) \frac{h(\tau) p(\tau)}{\gamma(\tau)}+\alpha h(\tau)-(t-\tau) h^{\prime}(\tau)\right]^{2} \\
& \leq(t-s)^{\alpha} h(s) \omega(s), \quad s \geq t_{0} .
\end{aligned}
$$

Divide (7) by $t^{\alpha}$ and take the upper limit as $t \rightarrow \infty$. Using (4), we obtain a contradiction. This completes the proof of the theorem.

REMARK 1. Note that Theorem 1 does not even require $\int_{t_{0}}^{\infty} d \tau / \gamma(\tau)=\infty$ as does the Wintner-Leighton theorem $[8,6]$, and does not require that the damping coefficient $p(t)$ is a "small" function.

REMARK 2. Theorem 1 above includes a result in [9] and Theorem 2 in [10] on equation (1) with $\gamma(t)=1$. [5].

The following corollary improves and generalizes Corollary 6 and Corollary 7 in

COROllary 1. If

$$
\begin{aligned}
\lim _{t \rightarrow \infty} \sup t^{-2} \int_{t_{0}}^{t}\left[(t-\tau)^{2} h(\tau) q(\tau)\right. & \\
& \left.-\frac{(t-\tau)^{2} h^{\prime 2}(\tau) \gamma(\tau)}{4 h(\tau)}-(t-\tau) h(\tau) \gamma^{\prime}(\tau)\right] d \tau=\infty
\end{aligned}
$$

with $h(t)$ as in Theorem 1 , then equation (**) is oscillatory.

ProOF. From (7), letting $p(t) \equiv 0$ and $\alpha=2$, we have that

(9)

$$
\begin{gathered}
t^{-2} \int_{t_{0}}^{t}\left[(t-\tau)^{2} h(\tau) q(\tau)-h(\tau) \gamma(\tau)-\frac{(t-\tau)^{2} h^{\prime 2}(\tau) \gamma(\tau)}{4 h(\tau)}+(t-\tau) h^{\prime}(\tau) \gamma(\tau)\right] d \tau \\
\leq t^{-2}\left(t-t_{0}\right)^{2} h\left(t_{0}\right) \omega\left(t_{0}\right)
\end{gathered}
$$


Since

$$
\begin{aligned}
\int_{t_{0}}^{t}(t-\tau) h^{\prime}(\tau) \gamma(\tau) d \tau=\int_{t_{0}}^{t} \int_{t_{0}}^{\tau} h^{\prime}(\xi) \gamma(\xi) d \xi d \tau \\
=\int_{t_{0}}^{t} h(\tau) \gamma(\tau) d \tau-\int_{t_{0}}^{t}(t-\tau) h(\tau) \gamma^{\prime}(\tau) d \tau-h\left(t_{0}\right) \gamma\left(t_{0}\right)\left(t-t_{0}\right)
\end{aligned}
$$

(9) and (10) together contradict our hypothesis (8).

THEOREM 2. Suppose that there exist a positive continuously differentiable function $h(t)$ on $\left[t_{0}, \infty\right)$ and $\alpha \in(1, \infty)$ such that

$$
\lim _{t \rightarrow \infty} \sup t^{-\alpha} \int_{t_{0}}^{t}(t-\tau)^{\alpha} h(\tau) q(\tau) d \tau<\infty,
$$

and there exists a continuous function $\varphi(t)$ on $\left[t_{0}, \infty\right)$ such that

$$
\begin{aligned}
\lim _{t \rightarrow \infty} \inf t^{-\alpha} \int_{0}^{t}\left\{(t-\tau)^{\alpha} h(\tau) q(\tau)\right. & \\
-\frac{1}{4}\left[(t-\tau) \frac{h(\tau) p(\tau)}{\gamma(\tau)}+\alpha h(\tau)-(t-\tau) h^{\prime}(\tau)\right]^{2} & \left.\times(t-\tau)^{\alpha_{2}} \frac{\gamma(\tau)}{h(\tau)}\right\} d \tau \geq \varphi(s)
\end{aligned}
$$

and

$$
\lim _{t \rightarrow \infty} \int_{t_{0}}^{t} \frac{\varphi_{+}^{2}(\tau)}{h(\tau) \gamma(\tau)} d \tau=\infty
$$

where $\varphi_{+}(t)=\max (\varphi(t), 0)$, then equation (1) is oscillatory.

PROOF. Suppose that $x(t)$ is a solution of (1) with $x(t) \neq 0$ for $t \geq t_{0}$. Set $\omega(t)=\gamma(t) x^{\prime}(t) / x(t)$. As in the proof of Theorem 1, (7) holds. Dividing (7) by $t^{\alpha}$ and taking the lower limit as $t \rightarrow \infty$, we obtain $\varphi(s) \leq h(s) \omega(s), s \geq t_{0}$, which implies that

$$
\varphi_{+}^{2}(s) \leq h^{2}(s) \omega^{2}(s) .
$$

We define functions

$$
\begin{array}{ll}
u(t)=t^{-\alpha} \int_{t_{0}}^{t}\left[(t-\tau) \frac{h(\tau) p(\tau)}{\gamma(\tau)}+\alpha h(\tau)-(t-\tau) h^{\prime}(\tau)\right](t-\tau)^{\alpha-1} \omega(\tau) d \tau, & t>t \\
v(t)=t^{-\alpha} \int_{t_{0}}^{t}(t-\tau)^{\alpha} h(\tau) \frac{\omega^{2}(\tau)}{\gamma(\tau)} d \tau, \quad t>t_{0} .
\end{array}
$$

From (6),

$$
u(t)+v(t)=t^{-\alpha}\left(t-t_{0}\right)^{\alpha} h\left(t_{0}\right) \omega\left(t_{0}\right)-t^{-\alpha} \int_{t_{0}}^{t}(t-\tau)^{\alpha} h(\tau) q(\tau) d \tau,
$$

and we observe that (12) implies that

$$
\lim _{t \rightarrow \infty} \inf t^{-\alpha} \int_{0}^{t}(t-\tau)^{\alpha} h(\tau) q(\tau) d \tau \geq \varphi(s), \quad s \geq t_{0},
$$


and

$$
\begin{array}{r}
\lim _{t \rightarrow \infty} \sup t^{-\alpha} \int_{t_{0}}^{t}(t-\tau)^{\alpha} h(\tau) q(\tau) d \tau \\
-\lim _{t \rightarrow \infty} \inf \frac{t^{-\alpha}}{4} \int_{t_{0}}^{t}\left[(t-\tau) \frac{h(\tau) p(\tau)}{\gamma(\tau)}+\alpha h(\tau)-(t-\tau) h(\tau)\right]^{2} \\
\quad \times(t-\tau)^{\alpha-2} \frac{\gamma(\tau)}{h(\tau)} d \tau \geq \varphi\left(t_{0}\right) .
\end{array}
$$

(17) together with (11) shows that there exists a sequence

$$
\left\{t_{n}\right\}_{1}^{\infty}, \quad t_{n}>t_{0}, \quad n=1,2,3, \ldots, \quad \lim _{n \rightarrow \infty} t_{n}=\infty
$$

such that

$$
\begin{aligned}
\lim _{n \rightarrow \infty} \frac{t_{n}^{-\alpha}}{4} \int_{t_{0}}^{t_{n}}\left[\left(t_{n}-\tau\right)\right. & \frac{h(\tau) p(\tau)}{\gamma(\tau)} \\
& \left.+\alpha h(\tau)-\left(t_{n}-\tau\right) h^{\prime}(\tau)\right]^{2}\left(t_{n}-\tau\right)^{\alpha-2} \frac{\gamma(\tau)}{h(\tau)} d \tau<\infty
\end{aligned}
$$

Taking the upper limit as $t \rightarrow \infty$ in (15) and using (16), we have

$$
\begin{aligned}
\lim _{t \rightarrow \infty} \sup \{u(t)+v(t)\} & =h\left(t_{0}\right) \omega\left(t_{0}\right)-\lim _{t \rightarrow \infty} \inf t^{-\alpha} \int_{t_{0}}^{t}(t-\tau) h(\tau) q(\tau) d \tau \\
& =h\left(t_{0}\right) \omega\left(t_{0}\right)-\varphi\left(t_{0}\right)=k
\end{aligned}
$$

Hence for all sufficiently large $n$,

$$
u\left(t_{n}\right)+v\left(t_{n}\right)<k .
$$

Since

$$
v(t)=\int_{t_{0}}^{t}\left(1-\frac{\tau}{t}\right)^{\alpha} h(\tau) \frac{\omega^{2}(\tau)}{\gamma(\tau)} d \tau>0
$$

is increasing in $t>t_{0}$, we see that $\lim _{t \rightarrow \infty} v(t)=c$, where $c=\infty$ or is a positive constant. Suppose that $c=\infty$, then $\lim _{n \rightarrow \infty} v\left(t_{n}\right)=\infty$ and, by (21),

$$
\lim _{n \rightarrow \infty} u\left(t_{n}\right)=-\infty \text {. }
$$

(21) and (22) lead to $u\left(t_{n}\right) / v\left(t_{n}\right)+1<\varepsilon$, where $0<\varepsilon<1$ is a constant, that is,

$$
u\left(t_{n}\right) / v\left(t_{n}\right)<\varepsilon-1<0, \text { for all large } t_{n} .
$$

One the other hand, by the Schwarz inequality we have

$$
\begin{aligned}
0 \leq & t_{n}^{-2 \alpha}\left(\int_{t_{0}}^{t_{n}}\left[\left(t_{n}-\tau\right) \frac{h(\tau) p(\tau)}{\gamma(\tau)}+\alpha h(\tau)-\left(t_{n}-\tau\right) h^{\prime}(\tau)\right]^{\alpha-1} \omega(\tau) d \tau\right)^{2} \\
\leq & \left(t_{n}^{-\alpha} \int_{t_{0}}^{t_{n}}\left[\left(t_{n}-\tau\right) \frac{h(\tau) p(\tau)}{\gamma(\tau)}+\alpha h(\tau)-\left(t_{n}-\tau\right) h^{\prime}(\tau)\right]^{2}\left(t_{n}-\tau\right)^{\alpha-2} \frac{\gamma(\tau)}{h(\tau)} d \tau\right) \\
& \cdot\left(t_{n}^{-\alpha} \int_{t_{0}}^{t_{n}}\left(t_{n}-\tau\right)^{\alpha} h(\tau) \frac{\omega^{2}(\tau)}{\gamma(\tau)} d \tau\right),
\end{aligned}
$$


for all large $t_{n}$, and so

$$
\begin{aligned}
0 & \leq \frac{u^{2}\left(t_{n}\right)}{v\left(t_{n}\right)} \\
& \leq t_{n}^{-\alpha} \int_{t_{0}}^{t_{n}}\left[\left(t_{n}-\tau\right) \frac{h(\tau) p(\tau)}{\gamma(\tau)}+\alpha h(\tau)-\left(t_{n}-\tau\right) h^{\prime}(\tau)\right]^{2}\left(t_{n}-\tau\right)^{\alpha-2} \frac{\gamma(\tau)}{h(\tau)} d \tau .
\end{aligned}
$$

By (19), we have

$$
\begin{aligned}
0 & \leq \lim _{n \rightarrow \infty} \frac{u^{2}\left(t_{n}\right)}{v\left(t_{n}\right)} \\
& \leq \lim _{n \rightarrow \infty} t_{n}^{-\alpha} \int_{t_{0}}^{t_{n}}\left[\left(t_{n}-\tau\right) \frac{h(\tau) p(\tau)}{\gamma(\tau)}+\alpha h(\tau)-\left(t_{n}-\tau\right) h^{\prime}(\tau)\right]\left(t_{n}-\tau\right)^{\alpha-2} \frac{\gamma(\tau)}{h(\tau)} d \tau \\
& <\infty .
\end{aligned}
$$

which contradicts (22) and (23).

Hence $\lim _{t \rightarrow \infty} v(t)=c<\infty$. Using (14), we then obtain that

$$
\begin{aligned}
\lim _{t \rightarrow \infty} t^{-\alpha} \int_{t_{0}}^{t}(t-\tau)^{\alpha} \frac{\varphi_{+}^{2}(\tau)}{h(\tau) \gamma(\tau)} d \tau \\
\leq \lim _{t \rightarrow \infty} t^{-\alpha} \int_{t_{0}}^{t}(t-\tau)^{\alpha} h(\tau) \frac{\omega^{2}(\tau)}{\gamma(\tau)} d \tau=c<\infty
\end{aligned}
$$

which contradicts condition (13). This completes the proof of Theorem 2.

REMARK 3. In the conditions of Theorem $2, q(t)$ is not required to be integrable or bounded on $\left[t_{0}, \infty\right)$. See Examples 2 and 3 below.

EXAMPLE 1. Consider the equation

$$
\left(\frac{1}{t} x^{\prime}(t)\right)^{\prime}+\sin t x^{\prime}(t)+t^{2} \cos t x(t)=0, \quad t \geq t_{0}>0 .
$$

If we take $h(t)=t$ and $\alpha=2$, then all the hypotheses of Theorem 1 are satisfied. Hence (24) is oscillatory, while oscillation criteria in [3,9 and 10] fail to apply to equation (24).

EXAMPLE 2. Consider the equation

$$
\left(t^{\lambda} x^{\prime}(t)\right)^{\prime}+t^{\mu} \sin t x^{\prime}(t)+t^{\nu} \cos t x(t)=0, \quad t \geq t_{0}>0
$$

where $-1 \leq \lambda<1,-\infty<\mu \leq-1$ and $-1<\nu \leq 1$ are constants and $2 \nu+1 \geq \lambda$. Taking $h(t)=1$ and $\alpha=2$, we have

$$
\begin{gathered}
\lim _{t \rightarrow \infty} \sup t^{-2} \int_{t_{0}}^{t}(t-\tau)^{2} \tau^{\nu} \cos \tau d \tau=-t_{0}^{\nu} \sin t_{0}<\infty \\
\lim _{t \rightarrow \infty} \inf t^{-2} \int_{s}^{t}\left\{(t-\tau)^{2} \tau^{\nu} \cos \tau d \tau-\frac{1}{4}\left[(t-\tau) \tau^{\mu} \sin \tau+2\right]^{2} \tau^{\lambda}\right\} d \tau \\
\geq-s^{\nu} \sin s-k, \quad s \geq t_{0},
\end{gathered}
$$


where $k$ is a positive constant. Set $\varphi(s)=-s^{\nu} \sin s-k$, there is an integer $N$ such that $(2 N+1) \pi+\pi / 4>t_{0}$, and when $n \geq N$ and $(2 n+1) \pi+\pi / 4 \leq s \leq$ $2(n+1) \pi-\pi / 4, \varphi(s)=-s^{\nu} \sin s-k \geq \varepsilon s^{\nu}$, where $\varepsilon$ is a small constant. Noting $2 \nu-\lambda \geq-1$, we have

$$
\begin{aligned}
\lim _{t \rightarrow \infty} \int_{t_{0}}^{t} \frac{\varphi_{+}^{2}(s)}{s^{\lambda}} d s & \geq \sum_{N=n}^{\infty} \varepsilon^{2} \int_{(2 n+1) \pi+\pi / 4}^{2(n+1) \pi-\pi / 4} s^{2 \nu-\lambda} d s \\
& \geq \sum_{N=n}^{\infty} \varepsilon^{2} \int_{(2 n+1) \pi+\pi / 4}^{2(n+1) \pi-\pi / 4} \frac{d s}{s}=\infty
\end{aligned}
$$

Hence (25) is oscillatory by Theorem 2, whereas known of the none criteria can cover this result.

ACKNOWLEDGMENT. The author wishes to thank the referee for a number of helpful and constructive comments. In particular he is indebted to the referee for his sugestions that lead to the present form of Theorem 2.

\section{REFERENCES}

1. W. J. Coles, An oscillation criterion for second-order differential equation, Proc. Amer. Math. Soc. 19 (1968), 755-759.

2. P. Hartman, On nonoscillatory linear differential equations of second order, Amer. J. Math. 74 (1952), 389-400.

3. I. V. Kamenev, Integral criterion for oscillations of linear differential equations of second order, Mat. Zametki 23 (1978), 249-251.

4. M. K. Kwong and A. Zettl, Asymptotically constant functions and second order oscillation, J. Math. Anal. Appl. 93 (1983), 475-494.

5. Integral inequalities and second order linear oscillation, J. Differential Equations 45 (1982), 16-33.

6. W. Leighton, On self-adjoint differential equations of second order, J. London Math. Soc. 27 (1952), 37-47.

7. D. Willett, On the oscillatory behavior of the solutions of second order linear differential equations, Ann. Polon. Math. 21 (1969), 175-194.

8. A. Wintner, A criterion of oscillatory stability, Quart. Appl. Math. 7 (1949), 115-117.

9. Jurang Yan, $A$ note on an oscillation criterion for an equation with damped term, Proc. Amer. Math. Soc. 90 (1984), 277-280.

10. C. C. Yeh, Oscillation theorems for nonlinear second order differential equations with damped term, Proc. Amer. Math. Soc. 84 (1982), 397-402.

Department of Mathematics, ShanXi University, Taiyuan, ShanXi, People's REPUBLIC OF CHINA 